\title{
Randomised trial of fluid restriction in ventilated very low birthweight infants
}

\author{
V Kavvadia, A Greenough, G Dimitriou, R Hooper
}

\begin{abstract}
Background-Fluid restriction has been reported to improve survival of infants without chronic lung disease (CLD), but it remains unknown whether it reduces CLD in a population at high risk of CLD routinely exposed to antenatal steroids and postnatal surfactant without increasing other adverse outcomes.

Aim-To investigate the impact of fluid restriction on the outcome of ventilated, very low birthweight infants.

Study design-A randomised trial of two fluid input levels in the perinatal period was performed. A total of 168 ventilated infants (median gestational age 27 weeks (range 23-33)) were randomly assigned to receive standard volumes of fluid (60 $\mathrm{ml} / \mathrm{kg}$ on day 1 progressing to $150 \mathrm{ml} / \mathrm{kg}$ on day 7 ) or be restricted to about $80 \%$ of standard input.

Results-Similar proportions of infants on the two regimens had CLD beyond 28 days $(56 \% \quad v 51 \%)$ and 36 weeks post conceptional age $(26 \% v 25 \%)$, survived without oxygen dependency at 28 days $(31 \% v 27 \%)$ and 36 weeks post conceptional age $(58 \% v 52 \%)$, and developed acute renal failure. There were no statistically significant differences between other outcomes, except that fewer of the restricted group ( $19 \% v 43 \%)$ required postnatal steroids $(p<0.01)$. In the trial population overall, duration of oxygen dependency related significantly to the colloid ( $p<0.01)$, but not crystalloid, input level; after adjustment for specified covariates, the hazard ratio was 1.07 (95\% confidence interval 1.02 to 1.13 ).

Conclusions-In ventilated, very low birthweight infants, fluid restriction in the perinatal period neither reduces CLD nor increases other adverse outcomes. Colloid infusion, however, is associated with increased duration of oxygen dependency. (Arch Dis Child Fetal Neonatal Ed 2000;83:F91-F96)
\end{abstract}

Keywords: chronic lung disease; prematurity; very low birthweight; fluid restriction; lungs; survival

High volumes of fluid input increase the likelihood of a patent ductus arteriosus (PDA), ${ }^{1}$ a known risk factor for the development of chronic lung disease (CLD). ${ }^{2}$ It is therefore surprising that fluid restriction in only one $\mathrm{e}^{3}$ of four ${ }^{13-5}$ randomised trials was associated with improved outcome. Interestingly, the positive effect was a lower mortality rather than a lower CLD rate. ${ }^{3}$ The population investigated were relatively mature and it is not clear how many were exposed to antenatal steroids or postnatal surfactant. ${ }^{3}$ Fluid restriction has a number of important possible side effects, including hypovolaemia which could result in renal impairment. We therefore felt it important to determine, in a high risk population, whether fluid restriction compared with a more liberal regimen was associated with a lower incidence of CLD but no increase in other adverse outcomes.

\section{Methods}

A randomised trial of two levels of fluid input was undertaken. Infants born prematurely with a birth weight of $\leqslant 1500$ g, without major congenital anomalies and requiring ventilation from within the first six hours of birth were eligible for entry into the study. If informed written consent was given by the parents, the infant was randomised to receive one of two fluid regimens by opening the next numbered and sealed envelope which contained details of the regimen the infant was to receive. Regimen A was similar to that advocated in a number of neonatal texts. ${ }^{67}$ Infants on regimen B (restricted) were to be prescribed at least $20 \%$ less fluid than those on regimen A (table 1); regimen $\mathrm{B}$ was similar to that previously used on our unit. Infants who were small for gestational age were started on the first day after birth on "day 2" of the respective fluid regimen and progressed accordingly. Fluid input on either regimen was only increased on the next day if the infant had lost weight and there were no signs of fluid overload, such as hyponatraemia (serum sodium $<135$ $\mathrm{mmol} / \mathrm{l}){ }^{8}$ The clinicians were allowed to deviate from the recommended fluid regimen if renal impairment, hypotension, or jaundice occurred. Infants who developed acute renal failure-that is, for any 24 hour period, creatinine levels $>132 \mu \mathrm{mol} / \mathrm{l}^{9}{ }^{10}$ and urine output $<1.0 \mathrm{ml} / \mathrm{kg} /$ hour, except on day 1 when the urine output had to be $<0.5$ $\mathrm{ml} / \mathrm{kg} /$ hour $^{11}$ - without evidence of dehydration were restricted to a fluid input equal to their urine output plus insensible losses. Infants who were hypotensive (mean blood pressure less than the 10th centile for their gestational age and birth weight ${ }^{12}$ ) were prescribed up to two boluses of colloid or crystalloid and then an inotrope infusion. Inotropes were used as first choice for blood pressure support if the infant was small for gestational age and had evidence of myocardial ischaemia. Infants who were jaundiced and required phototherapy were prescribed an extra $30 \mathrm{ml}$ fluid $/ \mathrm{kg} / 24$ hours. Infants who were hypoglycaemic (blood 
Table 1 Comparison of the recommended total fluid input on the two regimens

\begin{tabular}{lll}
\hline Day & Regimen $A$ & Regimen B (restricted) \\
\hline 1 & 70 & 40 \\
2 & 90 & $40-60$ \\
3 & 110 & 70 \\
4 & 120 & 90 \\
5 & 140 & 110 \\
6 & 150 & 130 \\
7 & 150 & 150 \\
\hline
\end{tabular}

Data are given as median or range $(\mathrm{ml} / \mathrm{kg})$.

glucose $<2.5 \mathrm{mmol} / \mathrm{l}$ ) were preferentially given an increased concentration of dextrose rather than an elevated volume of fluid. All fluid input (crystalloid and colloid) was recorded hourly and then totalled for each 24 hour period. To this was added the volume of all medication received by the infant. Urine was collected on open nappies or, for extremely low birthweight babies, on cotton wool balls placed on the nappy. As soon as the infant voided, the nappy or cotton wool ball was weighed to determine the amount of urine passed. If an infant was oliguric and the bladder palpable this was manually expressed. The urine output was totalled for each 24 hour period.

All infants were routinely monitored; this included at least daily assessment of electrolytes, creatinine, and bilirubin. No sodium supplements were given in the first 24 hours after birth, although the patency of indwelling arterial lines was maintained by infusion of heparinised $0.45 \%$ saline. Subsequently, sodium supplementation was altered to keep the serum sodium level between 135 and 145 $\mathrm{mmol} / 1 .{ }^{8}$ Blood pressure was measured once an hour, usually from intra-arterial monitoring. All infants were nursed in double walled closed incubators with $75-80 \%$ humidification, except in the first few hours when arterial and central lines were inserted. They were ventilated through shouldered oral endotracheal tubes using humidified gases. Enteral feeding $(0.5 \mathrm{ml} / \mathrm{h})$ was begun as soon as the clinical condition of the infant permitted. If enteral feeds were not tolerated, parenteral feeding (initially containing $0.5 \mathrm{~g}$ protein $/ \mathrm{kg} /$ day regardless of the volume of fluid infused) was started on day 4 .

The following were documented: duration of ventilation and oxygen dependency and whether the infants remained oxygen dependent at 28 days and/or 36 weeks post conceptional age, died, or developed other complications. An airleak was recorded as occurring if the infant had developed a pneumothorax or pulmonary interstitial emphysema. Regular cranial ultrasound examinations were performed to detect intracranial haemorrhage. A diagnosis of PDA was recorded if the clinical diagnosis was confirmed by an echocardiographic examination and either medical and/or surgical treatment was required. Infants with a proven PDA were fluid restricted and treated with indomethacin unless there was a contraindication (low platelets or renal failure). Surgical ligation was performed only if at least two courses of indomethacin had failed to close the PDA and the infant remained sympto- matic. Necrotising enterocolitis was diagnosed if the infant had abdominal distension and occult or gross blood in the stool with or without linear pneumatosis intestinalis on the abdominal $x$ ray picture.

Postnatal steroids (dexamethasone $0.5 \mathrm{mg}$ / $\mathrm{kg} /$ day for three days, $0.3 \mathrm{mg} / \mathrm{kg} /$ day for three days, and $0.1 \mathrm{mg} / \mathrm{kg} /$ day for three days) were prescribed if infants were considered to be at high risk of developing CLD - that is, they remained fully ventilator dependent beyond seven days or required at least $40 \%$ supplementary oxygen after three weeks, without signs of improvement over the subsequent 48 hours. Steroids were only administered once infection and a PDA had been excluded. Regular diuretic treatment (chlorothiazide and spironolactone) was prescribed after the first week of life for oxygen dependent infants in incipient right heart failure-that is, they showed rapid weight gain and evidence of oedema on the chest radiograph.

Infants were initially nursed in sufficient supplementary oxygen to keep their arterial oxygen tension between 45 and $80 \mathrm{~mm} \mathrm{Hg}$. Once continuous intra-arterial oxygen monitoring was no longer possible, oxygen saturation and transcutaneous monitoring was used in conjunction with intermittent arterial sampling. Supplementary oxygen was only discontinued when infants were able to keep their saturation levels above $95 \%$ throughout most of a 24 hour period; this was based on bedside nursing assessments.

\section{ANALYSIS}

The primary outcomes of the trial were survival without CLD and acute renal failure. The secondary outcomes were requirement for additional management strategies to improve respiratory status - that is, use of pancuronium, diuretics, steroids, inhaled nitric oxide, or high frequency oscillation-death before discharge, and the development of PDA, necrotising enterocolitis, airleak, or intracranial haemorrhage. Analysis was by intention to treat. Differences between the two groups were assessed for statistical significance using the $\chi^{2}$ or Fisher's exact test as appropriate. Duration of ventilation and oxygen dependency were compared between the two groups using log rank tests. Data from infants who died were censored. A secondary analysis using a Cox proportional hazards regression analysis was performed on both groups combined to assess the effects of total crystalloid and colloid input in the perinatal period on the duration of oxygen dependency. This led to results expressed in terms of hazard ratios, which show by how much the likelihood of an infant being oxygen dependent increased as a result of a given increase in total input.

SAMPLE SIZE

A retrospective notes audit disclosed that $22 \%$ of VLBW infants on our unit developed acute renal failure in the first 48 hours after birth and $62 \%$ survived without CLD. The infants had received a fluid input similar to regimen $B$ (restricted). We postulated that infants who 
Table 2 Comparison of the characteristics of the two groups

\begin{tabular}{|c|c|c|}
\hline & Regimen $A$ & $\begin{array}{l}\text { Regimen B } \\
\text { (restricted) }\end{array}$ \\
\hline Gestational age (weeks) & $27.5(23-33)$ & $27(23-33)$ \\
\hline Birth weight (g) & $914(486-1500)$ & $980(522-1499)$ \\
\hline Small for dates & $17(20)$ & $15(18)$ \\
\hline Caesarean section & $38(45)$ & $44(52)$ \\
\hline Male & $38(45)$ & $42(50)$ \\
\hline Antenatal steroids & $56(67)$ & $60(71)$ \\
\hline Surfactant & $57(68)$ & $60(71)$ \\
\hline \multicolumn{3}{|l|}{ In the first 48 hours } \\
\hline Max PIP $\left(\mathrm{cm} \mathrm{H}_{2} \mathrm{O}\right)$ & $19(12-40)$ & $20(14-42)$ \\
\hline $\mathrm{Max} \mathrm{FiO}_{2}$ & $0.63(0.21-1.00)$ & $0.65(0.21-1.00)$ \\
\hline
\end{tabular}

Data are given as median (range) or $\mathrm{n}(\%)$. The total number of infants in each group was 84 .

$\mathrm{FIO}_{2}$, fraction of inspired oxygen concentration; PIP, peak inspired pressure.

received a more liberal regimen (regimen $\mathrm{A}$ ) may have a higher rate of CLD and hence lower survival without CLD but would experience less renal impairment. Randomisation of 168 infants gave $80 \%$ power at the $5 \%$ level to detect a change in the likelihood of renal impairment from $22 \%$ to the $7 \%$ reported by others, ${ }^{13}$ and in survival without CLD from $62 \%$ to $42 \%$.

\section{Results}

The trial ran from April 1995 to October 1998, during which period there were 171 eligible babies. Two sets of parents did not give informed written consent and one unsupported mother was considered to be too psychiatrically unwell to be approached, consequently their three infants were not entered into the trial. The remaining 168 infants, median gestational age 27 weeks (range 23-33), were randomised to receive one or other of the two fluid regimens. Patients in the two groups had similar characteristics (table 2). In particular, the distributions of gestational ages and birth weights were similar between the two groups.

Although on each study day, a wide range of fluid inputs was actually received by infants on both regimens, infants on regimen $\mathrm{B}$ (restricted) received significantly $(p<0.01)$ less crystalloid than those on regimen $A$, up until day 7 , but similar levels of colloid (table 3 ). The usual colloid prescribed was albumin. In the first four days, infants on regimen $\mathrm{B}$ (restricted) received 19\% less fluid than those on regimen $\mathrm{A}$; overall the difference was $11 \%$. Infants on regimen $B$ had significantly lower urine outputs on days 2-6 inclusive (table 4). The proportions developing CLD or surviving without CLD did not differ significantly between the two groups (table 5). There were

Table 3 Comparison of the amount of fluid actually received on the two regimens

\begin{tabular}{llllll}
\hline & \multicolumn{2}{l}{ Regimen $A$} & & \multicolumn{2}{l}{ Regimen B (restricted) } \\
\cline { 2 - 3 } \cline { 5 - 6 } Day & Crystalloid & Colloid & & Crystalloid & Colloid \\
\hline 1 & $66[90.6](26-150)$ & $20(0-67)$ & & $55[76.0](26-129)$ & $11(0-102)$ \\
2 & $82[92.4](24-128)$ & $11(0-50)$ & & $70[82.1](30-147)$ & $9(0-63)$ \\
3 & $104[111.7](38-184)$ & $10(0-80)$ & & $84[98.6](43-233)$ & $0(0-65)$ \\
4 & $124[128.3](48-200)$ & $0(0-50)$ & & $103[112.9](30-171)$ & $0(0-47)$ \\
5 & $142[143.8](44-208)$ & $0(0-39)$ & & $121[125.8](53-182)$ & $0(0-57)$ \\
6 & $148[151.7](53-205)$ & $0(0-40)$ & & $136[140.4](49-204)$ & $0(0-40)$ \\
7 & $159[157.2](36-226)$ & $0(0-59)$ & & $146[149.0](54-198)$ & $0(0-51)$ \\
\hline
\end{tabular}

Data are given as median [mean] (range) $(\mathrm{ml} / \mathrm{kg})$.
Table 4 Comparison of the urine output (ml/kg/day) of the two groups

\begin{tabular}{llll}
\hline Day & Regimen $A$ & $\begin{array}{l}\text { Regimen } B \\
\text { (restricted) }\end{array}$ & p Value \\
\hline 1 & $35(0-188)$ & $31(0-139)$ & NS \\
2 & $96(0-185)$ & $78(0-204)$ & $<0.05$ \\
3 & $101(6-204)$ & $73(4-220)$ & $<0.001$ \\
4 & $109(31-178)$ & $82(14-174)$ & $<0.001$ \\
5 & $104(33-204)$ & $96(6-207)$ & $<0.01$ \\
6 & $108(48-193)$ & $93(16-192)$ & $<0.02$ \\
7 & $113(53-192)$ & $102(16-176)$ & NS
\end{tabular}

Data are given as median (range).

Table 5 Comparison of primary outcomes for the infants on the two regimens

\begin{tabular}{lll}
\hline & Regimen $A$ & $\begin{array}{l}\text { Regimen } B \\
\text { (restricted) }\end{array}$ \\
\hline $\mathrm{O}_{2}$ dependency at 28 days & $47(56)$ & $43(51)$ \\
$\mathrm{O}_{2}$ dependency at 36 weeks & $22(26)$ & $21(25)$ \\
Survivors without $\mathrm{O}_{2}$ dependency & & \\
At 28 days & $26(31)$ & $23(27)$ \\
At 36 weeks & $49(58)$ & $44(52)$ \\
Renal failure on days: & & \\
1 & $0[4,1]$ & $0[7,2]$ \\
2 & $0[2,2]$ & $1[7,5]$ \\
3 & $2[5,3]$ & $1[8,8]$ \\
4 & $0[5,3]$ & $1[9,8]$ \\
5 & $0[6,5]$ & $1[11,9]$ \\
6 & $0[5,5]$ & $1[14,10]$ \\
7 & $0[6,5]$ & $0[18,11]$ \\
\hline
\end{tabular}

Data are given as the number of affected individuals (\%). The total number in each group was 84 . Complete urine output or creatinine levels were not available on all the infants on all the study days. The initial number in the square brackets indicate the number of infants who had died and the second one the number for whom data were missing.

Table 6 Comparison of secondary outcomes for the infants on the two regimens

\begin{tabular}{lll}
\hline & Regimen $A$ & $\begin{array}{l}\text { Regimen } B \\
\text { (restricted) }\end{array}$ \\
\hline $\begin{array}{l}\text { Required treatment with } \\
\text { Pancuronium }\end{array}$ & $11 \%$ & $13 \%$ \\
Nitric oxide & $15(18)$ & $16(19)$ \\
HFO & $13(15)$ & $18(21)$ \\
Diuretics & $16(19)$ & $9(11)$ \\
Steroids & $36(43)$ & $16(19)$ \\
PDA & $22(26)[15] \dagger$ & $18(21)[16] \dagger$ \\
Airleak & $6(7)$ & $6(7)$ \\
ICH & $12(14)$ & $12(14)$ \\
NEC & $3(4)$ & $4(5)$ \\
Died & $16(19)$ & $21(25)$ \\
\hline
\end{tabular}

Data are expressed as the number (\%) of affected individuals. The total number in each group was 84 .

${ }^{\star}$ The number of ventilated days for which infants were paralysed is expressed as a percentage of the total number of ventilated days; data are given for day 1 only.

†The numbers in square brackets indicate the number of infants in each group who received indomethacin.

HFO, high frequency oscillation; PDA, patent ductus arteriosus; ICH, intracranial haemorrhage; NEC, necrotising enterocolitis.

also no significant differences in the proportion of infants in each group who developed renal failure. On each day there were a few missing data and infant deaths. Repeating the analysis using dummy variables for missing data and deaths also did not highlight a significant difference between the groups with regard to the incidence of renal failure on any of the study days (table 5 ). There were also no statistically significant differences between the two regimens with regard to occurrence of the secondary outcomes (table 6), except that more infants on regimen A required postnatal corticosteroids $(p<0.01)$. Steroids were prescribed for 52 infants; in 51 cases this treatment was started after the first week. The duration of ventilation (median 7 days (range 


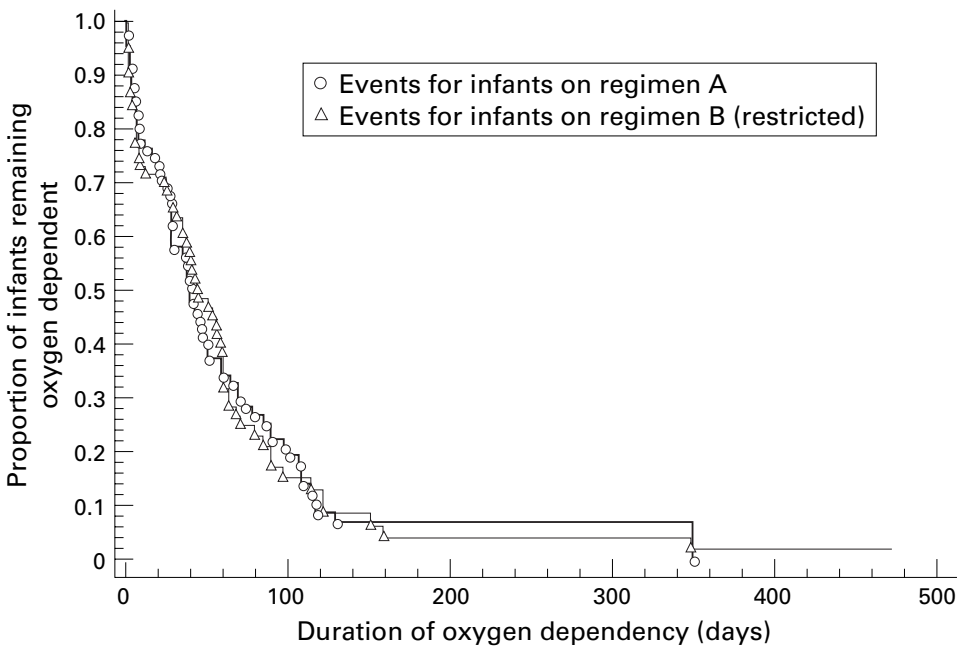

Figure 1 Kaplan-Meier curves of the proportion of infants remaining oxygen dependent during the first year of life. urine output is measured accurately. Neonates do not empty their bladders completely on voiding, and $7 \%$ fail to void during the first 24 hours of life, thus external urine collection of short duration is inaccurate. ${ }^{11}$ We measured the infants' urine output over each 24 hour period. The nurses observed the infants' open nappies for the passage of urine at least hourly. Once urine was voided, the nappy and/or cotton wool ball was weighed immediately, as delay could introduce inaccuracy because of evaporative loss. Data were missing from a minority of our patients, but treating those data and the data of infants who had died as dummy variables also did not show any statistically significant difference in the incidence of renal failure between the groups.

There are possible criticisms of our study. Firstly, it may be suggested that our fluid regimens were too similar. We would argue against that, as those on regimen B (restricted) were to receive at least $20 \%$ less fluid than those on regimen $\mathrm{A}$ and the latter regimen was similar to that recommended in standard texts. ${ }^{67}$ More stringent fluid restriction would probably increase the complication rate. ${ }^{4}$ Secondly, the trial regimens were only maintained during the first week. We felt that this was an appropriate duration, as it is in the first week that enhanced capillary permeability is present, and excess fluid is likely to leak into the pulmonary interstitium worsening lung function and predisposing the infant to CLD. Outside the perinatal period, fluid restriction could adversely impact on nutrition. Thirdly, we allowed the clinicians to alter the fluid input if this was dictated by a change in clinical condition-for example, the development of hypotension. As a consequence, the infants in both groups actually received a wide range of fluids on each study day. Nevertheless, those on regimen B (restricted) received significantly less fluid than those on regimen $\mathrm{A}$, as we had intended, and, during the first four days, $19 \%$ less fluid. There was, however, considerable overlap in the levels of fluid received by infants on the two regimens, but we do consider that our conclusion that fluid restriction does not reduce CLD is robust, as regression analysis of the data from the total study population confirmed that there was no significant relation between CLD and the level of crystalloid input. This result confirms earlier reports ${ }^{13-5}$ and, importantly, is generated from a population who were at high risk of CLD and routinely exposed to both antenatal steroids and postnatal surfactant.

We did not see any statistically significant differences in the mortality rate or occurrence of other adverse outcomes between our two groups. In earlier studies, an excess of PDA, ${ }^{1}$ necrotising enterocolitis, ${ }^{17}$ and mortality ${ }^{3}$ was reported to be associated with high, compared with low, fluid input levels. These studies, however, did not control for sodium intake. It has been previously shown ${ }^{18}$ that, if sodium intake is controlled, preterm infants are able to tolerate $200 \mathrm{ml} / \mathrm{kg}$ from the third day after birth, achieving a urine output of $7 \mathrm{ml} / \mathrm{kg} .{ }^{18}$ Our unit's routine policy was to avoid sodium supplementation in the first 24 hours and to tailor 
it subsequently, using the results of at least daily monitoring, to keep the levels within a predefined range. The infants on regimen $A$ had an intake recommended as standard ${ }^{67}$ rather than a "high" fluid intake, and it is the latter that has been linked with adverse outcome. ${ }^{1}$ Hence it is perhaps not surprising that we did not see an excess of adverse outcomes among infants on fluid regimen A. In addition, the significantly lower urine output of infants in the restricted group suggests that, within the range of fluids prescribed in this trial, they could compensate for the different intakes by altering their urine output. This may explain the lack of statistically significant differences in outcome relating to the magnitude of crystalloid intake.

Although the level of crystalloid input did not significantly affect CLD, the amount of colloid infused did. Colloid was prescribed to hypotensive infants, and thus an explanation for our findings could be that CLD develops in sicker infants. Interestingly, however, the occurrence of hypotension in the perinatal period did not differ significantly between the two groups ${ }^{19}$ and the association remained significant, although relatively small, after adjustment for factors known to influence the occurrence of CLD. It has been suggested that colloids do not increase the severity of pulmonary oedema in permeability states if equivalent filling pressures are used..$^{20}$ We have, however, previously shown that colloid administration adversely affects lung function in the perinatal period. ${ }^{21}$ A possible explanation is that colloid given in the perinatal period to infants with increased vascular permeability leaks into the lungs worsening respiratory function, ${ }^{21}$ necessitating higher levels of respiratory support and hence predisposing the infant to CLD. These results ${ }^{21}$ and the present ones further argue ${ }^{22} 23$ that clinicians should be extremely cautious about prescribing colloid infusions for critically ill patients.

A recent meta-analysis showed that, if steroids were started before 14 days of age, CLD was significantly reduced and, if they were started between 7 and 14 days, mortality was also lowered. ${ }^{24}$ The higher use of steroids for infants on regimen $\mathrm{A}$ is therefore likely to have influenced outcome and may explain why fluid restriction apparently did not reduce the occurrence of CLD. The clinicians who prescribed the steroids were not blinded to the infant's fluid regimen, but for most cases began treatment outside the perinatal period. Before the start of the trial, the routine policy of the unit was to use the more restricted fluid regimen. We do not feel, however, that this biased the clinicians with regard to prescription of steroids, as we have a clear protocol for this. Thus the greater use of steroids for the group on regimen A suggests that the clinicians assessed them to have more severe lung disease and hence to be at greater risk of developing CLD. Corticosteroids have many acute side effects and there is increasing concern that they may also have long term adverse consequences, ${ }^{25}$ including neuromotor dysfunction $^{26}$ and abnormal lung growth and

\section{Key messages}

- High fluid volumes increase the likelihood of a PDA, a risk factor for CLD development

- One of four previous randomised trials showed that fluid restriction improved outcome - that is, a lower mortality in a relatively mature population

- We now show in ventilated VLBW infants that fluid restriction in the perinatal period does not reduce CLD; colloid infusion, however, increases duration of oxygen dependency

development. ${ }^{27-29}$ As it was associated with a lower requirement for postnatal steroids, fluid restriction may therefore be advantageous for the VLBW infant.

We conclude that fluid restriction to less than $90 \%$ of usual maintenance values ${ }^{67}$ neither reduces CLD nor increases other adverse outcomes. Colloid infusion in the perinatal period, however, may impair long term lung function. V K was supported by the South Thames Regional Health
Authority Research and Development Directorate and G D by Authority Research and Development Directorate and G D by
the Children Nationwide/Nestle' Research Fellowship. We the Children Nationwide/Nestle Research
thank Ms Sue Williams for secretarial support.

\section{Appendix}

Infants received surfactant (Survanta) if they were ventilated in at least $30 \%$ oxygen. The surfactant was administered within two hours of birth; a second dose was given 12 hours later if the infant remained ventilator dependent and still required supplementary oxygen. Infants were initially supported on conventional ventilation at rates of $60-120$ breaths/min and inspiratory to expiratory ratios of $1: 1.2$. Pancuronium was only administered if, after rate and inspiratory time manipulation, the infant continued to actively expire and therefore to be at high risk of developing a pneumothorax. Infants who, after the second dose of surfactant, required a mean airway pressure of at least $12 \mathrm{~cm} \mathrm{H}_{2} \mathrm{O}$ and an inspired oxygen concentration of $50 \%$ were transferred to high frequency oscillation; a high volume strategy was used. Infants with pulmonary hypertension were given inhaled nitric oxide, provided that they had no bleeding tendency, a platelet count $>100000 / \mu 1$, and no greater than a grade II intracranial haemorrhage.

1 Bell EF, Warburton D, Stonestreet B, Oh W. Effect of fluid administration on the development of symptomatic patent ductus arteriosus and congestive heart failure in premature infants. N Engl f Med 1980;302:598-604.

2 Rojas MA, Gonzalez A, Bancalari E, Claure N, Poole C, Silva-Neto G. Changing trends in the epidemiology and Silvalogy and pathogenesis of neon

3 Tammela OKT, Kovisto ME. Fluid restriction for preventing bronchopulmonary dysplasia? Reduced fluid intake during the first weeks of life improves the outcome intake during the first weeks of life improves the outcome
oflow birthweight infants. Acta Paediatr Scand oflow birthweight

4 Lorenz JM, Kleinman LI, Kotagal UR, Reller MD. Water balance in very low birthweight infants: relationship to water and sodium intake and effect on outcome. $\mathcal{F}$ Pediatr 1982;101:423-32.

5 von Stockhausen HB, Struve M. Die auswirkungen einer stark unterschiedlichen parenteralen Fluessigkertszufuhr bei Frueh- und Neugeborenen in den erstein drei Lebenstagen. Klin Padiatr 1980;192:539-46.

6 Black JA, Whitfield MF, eds. Neonatal emergencies. London: Butterworth, 1991.

7 Roberton NRC, ed. Manual of neonatal intensive care. 3rd ed. London: Edward Arnold, 1993.

8 Shaffer SG, Weismann DN. Fluid requirements in the preterm infant. Clin Perinatol 1992;19:233-50.

9 Chevalier RL, Campbell F, Brenbridge ANAG. Prognostic factors in acute renal failure. Pediatrics 1984;74:265-72. 
10 Stapleton FB, Jones DP, Green RS. Acute renal failure in neonates: incidence, aetiology and outcome. Pediatr Nephrol 1987;1:314-20.

11 Modi N. Sodium intake and preterm babies. Arch Dis Child 1993;69:87-91

12 Watkins AMC, West CR, Cooke RWI. Blood pressure and cerebral haemorrhage and ischaemia in very low birthweight infants. Early Hum Dev 1989;19:103-10.

13 Guignard JP, Tarado A, Mazouni SM, Gautier E. Renal function in respiratory distress syndrome. $f$ Pediatr 1976;88:845.

14 Palta M, Sadek M, Barnet JH, et al for the Newborn Lung Project. Evaluation of criteria for chronic lung disease in surviving very low birthweight infants. $f$ Pediatr 1998;132:57-63.

15 Kinali M, Greenough A, Dimitriou G, Yuksel B. Chronic respiratory morbidity following premature delivery: prediction of prolonged respiratory support requirement. Eur f Pediatr 1999;158:493-6.

16 Miall LS, Henderson MJ, Turner AJ, et al. Plasma creatinine rises dramatically in the first 48 hours of life in preterm infants. Pediatrics 1999;104: 76 .

17 Bell EF, Warburton D, Stonestreet BS, Oh W. High volume fluid intake predisposes premature infants to necrotising enterocolitis. Lancet 1979;88:90.

18 Leake RD, Zakauddin S, Trygstad CW, Fu P, Oh W. The effects of large volume intravenous fluid infusion on neonatal renal function. F Pediatr 1976;89:968-72.

19 Kavvadia V, Greenough A, Dimitriou G, Forsling M. Randomized trial of two levels of fluid input in the perinatal period. Effect on fluid balance, electrolyte and metabolic disturbances in ventilated very low birthweight infants. Acta Paediatr 2000; in press.
20 Metildi L, Shackford S, Virgilo R, Peters RM. Crystalloid versus colloid in resuscitation of patients with severe pulmonary insufficiency. Surgery in Gynecology and Obstetrics 1984;158:207-12.

21 Kavvadia V, Greenough A, Dimitriou G, Hooper R. Comparison of the effect of two fluid input regimens on perinatal lung function in ventilated very low birthweight infants. Eur f Pediatr 1999;158:917-22.

22 Greenough A. Use and misuse of albumin infusions in neonatal care. Eur f Pediatr 1999; 157:699-702.

23 Schierhout G, Roberts I. Fluid resuscitation with colloid or crystalloid solutions in critically ill patients: a systematic review of randomised trials. BMF 1998;316:961-4.

24 Bhuta T, Ohlsson A. Systematic review and meta-analysis of early postnatal dexamethasone for prevention of chronic lung disease. Arch Dis Child Fetal Neonatal Ed 1998;79:F26-33.

25 Greenough A. Gains and losses from dexamethasone for neonatal chronic lung disease. Lancet 1998;352:835-6.

26 Yeh TF, Lin YJ, Huang CC, et al. Early dexamethasone therapy in preterm infants: a follow-up study. Pediatrics 1998;101:e7.

27 Massaro D, Massaro GD. Dexamethasone accelerates postnatal alveolar wall thinning and alters wall composition. Am f Physiol 1986;251:R218-24.

28 Sahe J, Ami H, Domino M. Effects of postnatal dexamethasone treatment on development of alveoli in adult rats. Exp Lung Res 1989;15:961-73.

29 Tschanz SA, Damke BM, Burri PH. Influence of postnatally administered glucocorticoids on rat lung growth. Biol Neonate 1995;68:229-45. 